\title{
Teachers matter for metacognition: facilitating metacognition in the primary school through teacher-pupil interactions
}

\author{
Heather E. Branigan, and David I. Donaldson
}

\begin{tabular}{|l|l|}
\hline Date of deposit & 10 December 2020 \\
\hline Document version & Author's accepted manuscript \\
\hline Access rights & $\begin{array}{l}\text { Copyright C } 2020 \text { Elsevier Ltd. All rights reserved. All rights } \\
\text { reserved. This work is made available online in accordance with } \\
\text { the publisher's policies. This is the author created, accepted } \\
\text { version manuscript following peer review and may differ slightly } \\
\text { from the final published version. }\end{array}$ \\
\hline $\begin{array}{l}\text { Citation for } \\
\text { published version }\end{array}$ & $\begin{array}{l}\text { Branigan, H. E., \& Donaldson, D. I. (2020). Teachers matter for } \\
\text { metacognition: facilitating metacognition in the primary school } \\
\text { through teacher-pupil interactions. Thinking Skills and Creativity, } \\
38,[100718] .\end{array}$ \\
\hline $\begin{array}{l}\text { Link to published } \\
\text { version }\end{array}$ & \begin{tabular}{l} 
https://doi.org/10.1016/j.tsc.2020.100718 \\
\hline
\end{tabular}
\end{tabular}

Full metadata for this item is available in St Andrews Research

Repository at: https://research-repository.st-andrews.ac.uk/

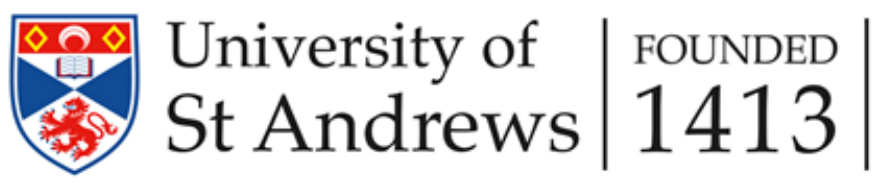




\title{
Teachers matter for metacognition: Facilitating metacognition in the primary school through teacher-pupil interactions
}

\begin{abstract}
Despite the ubiquity of Structured Thinking Activities (STAs) throughout primary school education in the UK and beyond, little is known about the ways that activities such as learning logs are used to support pupils to think about and manage their own thinking (i.e., engage with metacognition). Here we investigated how pupils engaged with STAs throughout a school year by conducting an in-depth case study of one Scottish primary four classroom, examining factors that facilitated and/or inhibited pupil metacognition. By triangulating data from participant observation, interviews and document analysis, we found that pupils were often un-motivated to engage with STAs, with responses provided during written and oral activities typically revealing superficial references to classroom topics of interest. Whilst factors such as the classroom culture and the timing of activities were found to influence pupil engagement with STAs, observational data indicated that teacher-pupil interactions are essential for eliciting metacognition from pupils (i.e., via discussions that occurred as part of the STAs). Our findings suggest that teachers play a critical role in encouraging elaboration from pupils in relation to descriptions of their own thinking and learning, particularly when pupils' initial responses are broad or superficial. We discuss the critical importance of teacher talk for metacognition, emphasising the 'dual role' that teachers must play when facilitating metacognition within the classroom.
\end{abstract}

Keywords: metacognition; teacher-pupil interactions; learning logs; pedagogy 


\section{Teachers matter for metacognition: Facilitating metacognition in the primary school through teacher-pupil interactions.}

Structured Thinking Activities (STAs) such as learning logs, learning diaries or achievement logs are used routinely throughout Scottish primary education and beyond (e.g., in the USA; Hurst, 2005). Indeed, the use of STAs is widely viewed as good practice (e.g., Kidner, 2013), with teachers adopting a variety of STAs to support pupil-led learning, share successes and provide a systematic platform to encourage pupils to think about their thinking (i.e., to engage in metacognition; Authors, 2019; Higgins et al., 2006). Despite the ubiquity of STAs in Scottish education, little is known about how STAs are actually used in primary school classrooms as pedagogical tools. Equally, it is unclear how teachers and pupils interact with STAs to support the development of metacognition. As such, the present study aims to develop understanding about the ways that STAs are used within the classroom, and crucially, what factors facilitate or inhibit metacognition through STAs.

\section{Defining STAs}

In the present study, we use the term Structured Thinking Activities (hereafter STAs) to refer to specific pedagogical tools that are used within the classroom with the explicit aim of encouraging pupils to think about and/or manage their own thinking. Importantly, we distinguish between STAs and everyday classroom activities that may include an aspect of thinking about thinking, but where thinking about thinking itself is not the sole goal of the task (Barclay, 1996; Moon, 2002). From this perspective STAs can be distinguished from other tasks (such as numeracy or literacy), due to the explicit focus of STAs upon the processes of thinking and learning. In practice, STAs are often presented in the form of weekly or termly tasks (such as planning or reflection activities). Researchers originating from the NorthEast Thinking Skills Network draw a similar distinction between pedagogical practices embedded throughout everyday classroom activities and more structured approaches, often under the broad headings of 'thinking skills' and 'learning to learn' (e.g., see Higgins et al., 2007).

Common STAs used in educational practice include learning journals, learning logs, planning books and achievement logs (Moon, 2002). Other STAs that have been documented in research include 'thoughts and feelings books' to document weekly reflections on learning, 'pupil response logs' to provide teacher feedback as well as pupil reflection, and 'pupil learner log books' to encourage reflection (Higgins et al., 2006; Wall \& Hall, 2016). Pupil Views Templates (Wall, 2008; Wall, Higgins, Remedios, Rafferty \& Tiplady, 2012) and concept maps (Ritchhart, Turner \& Hadar, 2009) are other examples of structured activities that have been developed to elicit children's understanding of their own thinking and learning in the classroom. Pupil View Templates (PVTs) are visual tools that enable pupils to describe their thinking and/or learning in a specific scenario, utilising visual prompts such as empty thought or speech bubbles (Wall \& Higgins, 2006; Wall et al., 2012). A benefit of activities such as PVTs is that they can be considered both as pedagogical tools (to support metacognition), and as research tools (to investigate metacognition; Wall \& Higgins, 2006; Wall, 2008). Moreover, PVTs and concept maps can be used in discussion with pupils, as tools that encourage pupils to provide reflections on their learning in specific contexts (Wall et al., 2012). As such, a commonality across different STAs is that whilst they all involve some form of written output, STAs are not confined to written activities in isolation and are typically used alongside discussion between pupils and teachers.

Existing research suggests that STAs are associated with a range of benefits for learners, including demanding time and intellectual space; promoting independent learning; providing 
a focusing point to order thinking; supporting emotional or affective components of learning; and assisting with non-straightforward aspects of learning (Moon, 2002). More importantly for present purposes, within primary schools recent survey evidence suggests that STAs are one of the main routes used by teachers to encourage children to think about and manage their own thinking (i.e., to engage with metacognition; Author, 2019).

\section{Metacognition and STAs}

In broad terms, metacognition is described as the ability to think about and manage one's own thinking. One of the key theorists in the field of metacognition is John Flavell, who defined metacognition as "knowledge and cognition about cognitive phenomena" (Flavell, 1979, p906). Accounts of metacognition broadly differentiate between two main components; metacognitive knowledge (i.e., 'thinking about thinking', for example, knowledge of persons, tasks and strategies; Flavell, 1979) and metacognitive regulation (i.e., managing thinking, for example, controlling cognition through planning, monitoring and evaluating; Brown, 1987; Schraw \& Moshman, 1995). Indeed, Flavell (1976) defined metacognition as encapsulating both knowledge and regulation of cognition: "Metacognition refers, among other things, to the active monitoring and consequent regulation and orchestration of these processes in relation to the cognitive objects or data on which they bear" (Flavell, 1976, p232). This twocomponent definition has been further divided into different sub-components by Flavell (1979), as well as Schraw and Moshman (1995). To gain more clarity about sub-components of metacognition (including aspects of knowledge, monitoring and evaluation), Scott and Levy (2013) conducted exploratory factor analysis on a metacognitive questionnaire. Despite the multi-faceted nature of definitions of metacognition across the field, factor analysis produced two clear components: knowledge and regulation.

Within the educational context, metacognition has been related to effective studying and learning, with research clearly demonstrating the beneficial effects of metacognition for educational success (for review see Dignath, Buettner \& Landgfeldt, 2008). Consistent with this view, the Education Endowment Foundation highlighted metacognition and selfregulation approaches as leading to an average of seven months additional progress (Higgins et al., 2016).

The primary school years have been highlighted as a particularly critical time for the development of metacognition (Roebers, 2014). Whilst indicators of metacognition have been identified in observational studies of children from the early years (Robson, 2010, 2015), findings from cognitive psychological research suggest that children become more skilled in estimating their own performance and controlling their cognition as they reach the age of between 9 and 12 years old (Roebers, Schmidt \& Roderer, 2009). Most importantly for educational practice, research consistently demonstrates that teachers can play a key role in promoting metacognition in the classroom. For instance, research has highlighted the key importance of teachers adopting appropriate talk about thinking and learning (Robson, 2010; Hacker \& Dunlosky, 2003), as well as providing instrumental support that provides just enough scaffolding for students to perform a task independently (Perry, 1998; Perry et al., 2002). Moreover, in their meta-analysis of thinking skills initiatives, Higgins et al. (2004) found that initiatives were most successful when they were explicitly instructed, with discussion also playing a key role in making the thinking process explicit to students. In short, therefore, a considerable body of research has identified metacognition as clearly beneficial for learning, with teachers playing a key role for promoting metacognition throughout the primary school years. 
One approach to facilitate metacognition in the classroom is through specific structured thinking activities. Indeed, the relationship between STAs and metacognition is intuitive - the use of logs and journals is clearly intended to encourage pupils to think about their thinking and reflect on learning in relation to past and future lessons. Links between STAs and improved metacognition have also been demonstrated within high school and further education contexts. For example, Audet et al. (1996) found that learning logs supported high school students' understanding of scientific concepts. Similarly, McCrindle and Christensen (1995) found that university students who documented their learning in journals had increased metacognition, cognitive strategy use, and science performance compared to controls who completed a scientific report. Also, in higher education, Nückles, Hübner and Renkel (2009) found that cognitive performance was improved when students were given cognitive and metacognitive prompts in learning journals (compared to no prompts).

In a study that investigated the use of interactive student notebooks as an STA in science lessons, Mallozzi and Heilbronner (2013) found that $7^{\text {th }}$ grade (aged 12-13 year-old) pupils' learning was enhanced through STAs. More specifically, the use of STAs was found to encourage pupils to use metacognitive strategies, and to use reflection to develop a sense of personal ownership of their learning. Similarly, Smith, Rook and Smith (2007), demonstrated the educational benefits of STAs by examining the influence of different types of questioning (through journals) upon high school pupils' academic performance. Overall, findings suggest that engaging with journal activities improved pupil grades (compared to no journal writing). Interestingly, questions focusing on metacognitive and affective aspects of learning led to greater improvement than purely cognitive questions. Clearly, therefore, there is evidence to suggest a positive relationship between STAs and metacognition within the classroom.

\section{STAs as pedagogical tools}

The evidence outlined above highlights just how important engagement with the content of STAs is for their success. By contrast, however, existing studies have provided relatively little insight into the importance of the context in which STAs are used. To some degree this appears to reflect the belief that the provision of STAs will in itself be sufficient to improve academic performance. As a result, despite the clear prominence of STAs in education and research suggesting beneficial effects upon both learning and metacognition, there is a lack of research (particularly within the psychological field) that investigates the ways that STAs are actually used within educational settings (Paris \& Paris, 2001).

It is important to note that whilst there is limited psychological research investigating the relationship between STAs and metacognition in applied settings, there is a considerable body of evidence to suggest that STAs are powerful pedagogical tools to support thinking skills and metacognition. For instance, in a review of 'thinking skills' programmes throughout formal schooling, Higgins et al. (2004) identified seventeen specific programmes used in classrooms. Furthermore, a review of professional enquiry approaches used in English schools identified a diverse range of approaches to encourage thinking skills, under the ' 5 Rs' of Resilience, Readiness, Reflectiveness, Resourcefulness and Remembering (Higgins et al., 2007). In particular, classroom-based research has identified PVTs as powerful tools for eliciting metacognition (Wall \& Higgins, 2006), particularly when incorporated within wider pedagogies such as discussion (Wall et al., 2012). Again, from this perspective there is little doubt that STAs can be effective, but further research is required to explore the use of STAs in classrooms, and critically, the mechanisms through which they facilitate metacognition. 
A related issue that has received little attention in the literature is the key factors in the classroom that might facilitate or inhibit the use of STAs. For example, teachers clearly play a role in facilitating STAs, as highlighted by Barclay (1996, p41): 'it is important [...] that learning logs should not be given to learners and just "left to get on with it". It is a developmental technique which requires good support'. Furthermore, the role of the teacher in providing opportunities for students to independently think about their thinking through STAs has been emphasised by Kleinsasser and Horsch (1992, p15): 'when teachers relinquish some control of what is learned and how to learn it and when students are allowed to monitor their own progress, learning is enhanced and accelerated'. Given the vital role that teachers play in supporting the development of pupil metacognition (Hacker \& Dunlosky, 2003; Perry et al., 2002; Robson, 2010), the lack of research regarding the role of STAs within teaching practice is surprising. Clearly, one important component concerns how STAs are used in classrooms - what output is generated using STAs, how do teachers support pupils, and how does the use of STAs facilitate metacognition?

The present study

Given the prominence of STAs within primary education there is a surprising lack of research investigating the use of STAs as everyday pedagogical tools within the classroom. Moreover, given the apparent relationship between STAs and metacognition, there is a lack of understanding about what factors facilitate and/or inhibit metacognition through STAs, particularly in relation to the role of the teacher in structuring the activities themselves. In the present study, therefore, we aim to investigate the use of one specific STA within primary school education, and to examine the factors that facilitate and/or inhibit pupils' engagement with the STAs and (ultimately) influence metacognition.

In the present study, we join a primary four classroom as they are introduced to STAs and follow the class as they negotiate the use of STAs throughout one school year. This case study approach provides us with an opportunity to develop rich understanding about pupils' use of STAs, and importantly, factors within the classroom that influence pupils' metacognition throughout STAs. The focus on STAs as pedagogical tools allows us to build on our exploration of pupils' experiences with STAs, as detailed in Authors (2019). We are guided by two broad questions: In what ways do pupils think about and manage their own thinking through STAs? And, what factors in the learning and teaching environment of the classroom facilitate and/or inhibit pupils' metacognition through the use of STAs?

\section{Methodology}

The present study adopted a case study approach to investigate STAs within one primary four classroom in Scotland throughout a school year (September 2016 - June 2017). With a focus on STAs, we drew on ethnographic methodologies to provide rich descriptive data regarding the use of STAs in the classroom context (Geertz, 1973; Robson, 2010). In the present study, we triangulated data by conducting participant observation, interviews and analysis of written texts. Observations were conducted throughout the entirety of STA sessions (i.e., from when the activity was presented to pupils until the activity was ended by the teacher - typically at the end of the school day). Observations were documented in field notes, using real-time narrative running records (Perry, 1998). A benefit of observations is their relatively unobtrusive nature (Adler \& Adler, 1998). Moreover, observations facilitate investigation of external factors in the classroom that might influence student metacognition, such as interactions with the teacher or peers. Of course, metacognition is an internal process and so can be difficult to observe (Audet et al., 1996; Wall, 2008). As such, short semi-and un- 
structured discussions with teacher and students were conducted throughout the observation period, adding depth to the data collected for each task (Lewis \& Porter, 2007). The final source of data collected in the present research was the written excerpts of students' STAs. The primary use of such visual data was further triangulation of findings, providing additional 'illuminative' evidence in addition to interviews and observations by comparing the 'products' to the 'process' of STAs (Craft, Cremin, Hay \& Clack, 2014; Hammersley, 2006).

Our analysis was driven by our research questions, which focused on the ways that learners engage with STAs, and the classroom factors that facilitate and/or inhibit metacognition through STAs. As such, the findings presented in this study reflect our distinctive interdisciplinary approach to thematic analysis. That is, this study was interpretive in design and used inductive thematic analysis that maintained a firm grounding in practice, whilst being clearly guided by psychological theory. The broadly interpretivist case study design provided the opportunity to investigate the interactions between pupils and their teacher, providing deeper insight about the metacognitive process surrounding STAs in primary schools. Analysis consisted of the following procedures (drawing from the 'phases of thematic analysis' by Braun \& Clarke, 2006): Familiarisation with the data, generation of initial codes and themes, naming and defining themes, review of themes and writing up. Full details of procedures employed for data collection and analysis are provided in Authors (2019).

\section{Structured Thinking Activities at Forestview Primary School}

The research took place within Forestview Primary School, a non-denominational local authority school in central Scotland. The specific classroom that formed the basis of our case study was Ms Abbot's primary four class, which comprised of pupils aged between seven and eight years old. On beginning the school year, Ms Abbot had been a qualified teacher for four years. In total, data collection was conducted throughout nine hours, observing STAs throughout the course of the school year, over nine data collection episodes (averaging 60 minutes, ranging 45-105 minutes).

\section{Class Meets and Achievement Logs}

Within Ms Abbot's primary four class, both weekly and termly STAs were routinely implemented, using learning logs and achievement logs respectively. The classroom researcher (first author) joined the class as weekly STAs were initially introduced to pupils, in the form of 'class meets' and associated written learning logs that were completed on Friday afternoons. During class meets, Ms Abbot brought pupils together as a group, at the front of the class, and discussed pupils' learning from throughout the week. Discussions were structured around three sentences that were presented on the whiteboard. Examples of sentences included were:

- This week I have enjoyed...

- Something I've found tricky this week has been...

- It will help me next week if I...

Ms Abbot discussed sentences with pupils as they were grouped together. Following discussion, pupils were often asked to return to their desks and complete the sentences in their 'learning logs'. As described by Ms Abbot to pupils at the beginning of the school year, Learning logs were weekly STAs that were provided to each pupil to reflect on their thinking and learning from throughout the week by completing sentences set by Ms Abbot; 
It's just for you to reflect on your week. It's all about what's happened in the week, and what you can do next week to keep improving.

In comparison to the newly-introduced learning logs, achievement logs were an established STA throughout Forestview primary school when the research study began. Achievement logs were used in all classrooms to encourage pupils to set targets for each term in the school calendar (for example by thinking about next steps for learning), and to reflect on their targets at the end of the terms (for example by 'using 'traffic lights' to evaluate progress and comparing to a teacher evaluations). Example achievement log entries are provided in Figures 1 and 2. Similar to learning logs, achievement logs were typically paired with a class discussion, in which Ms Abbot brought pupils together as a group and discussed pupils' learning for the term to come (at the start of term) or reflected on learning (at the end of term). In addition to supporting planning and reflection, achievement logs were also used as a platform for celebrating successes and demonstrating achievements. Achievement logs were kept by the teacher within the classroom, but were also taken home by pupils once a term to share the logs with parents/guardians (who were invited to comment).

[Figure 1 about here]

[Figure 2 about here]

\section{Findings}

As stated in the introduction, we were guided by research questions concerning the ways that pupils think about and manage their own thinking through STAs, and specifically, the factors in the learning and teaching environment of the classroom that facilitate and/or inhibit pupils' metacognition. Consequently, in the following section, we present findings that highlight factors within the classroom that we identified as influencing pupils' engagement with STAs. Throughout the presentation of findings, we draw on excerpts from both observations and interviews, with indicative evidence from written outputs also presented. Responses provided by pupils are noted in squared parentheses.

Creating an environment that supports thinking about thinking

From the beginning of data collection, it was clear that Ms Abbot explicitly valued pupils being able to discuss their thinking and learning in a supportive environment. In observed teacher-pupil discussions surrounding weekly learning log activities, Ms Abbot repeatedly reiterated that pupils are individual and unique in how they learn, without singling out any one pupil in a manner that might cause embarrassment. For example, when a pupil stated that they prefer to learn in a quiet environment, Ms Abbot drew a comparison with another pupil in the class;

It's interesting, because Laura liked it when she was up and doing the active things this morning [...] do we all learn in the same way? [pupils shout no].

In this excerpt, Ms Abbot's focus on interesting differences created an environment that encouraged pupils to openly explore differences between them and start to think about differences in thinking in particular. Later in the year, Ms Abbot continued to refer to differences as being natural;

Some people like doing things in different ways [...] it doesn't mean that one way is right and one's wrong. It just means that we like doing things in different ways. 
Ms Abbot created a culture of mutual respect for differences in learning through her discussions with the class as part of STAs. Thus, the culture created in the classroom was one that placed value upon pupils being able to openly think about their thinking (i.e., engage with metacognition).

A culture that valued thinking and learning was supported by the growth mindsets approach in the classroom, encouraging and celebrating resilience and persistence. In one observed teacher-pupil discussion, Ms Abbot asked pupils to reflect on the statement 'this week I am proud of myself for...'. In discussion about a pupils' response of times tables, Ms Abbot stated;

You should all be proud of yourselves for the work you put in to learning your times tables. And you know what it was? Remember the growth mindsets and we said that you're not just born knowing things, we have to work hard to learn them?

In this excerpt, Ms Abbot clearly praised pupils for their efforts in the subject and linked pupils' responses to the growth mindset approach. Ms Abbott also discussed the positive feelings that result from persistence;

Do you ever get that feeling when something is hard, and you want to give up, and you don't give up? And after you've done it you just feel so good about yourself, like yes, I'm on fire, I just feel so good about this.

Here we can see that through discussion drawing on the growth mindset approach, Ms Abbot explicitly discussed with pupils that it is important to persist in the face of struggle. Across the observation periods, we found that a focus on growth mindsets and persistence were factors of classroom practice that positively influenced classroom culture.

Another key theme in relation to supporting pupils' engagement with their own learning throughout STAs was the focus upon pupil-led learning. Though unstructured interview between researcher and Ms Abbot, it became clear that she saw pupil-led learning as a route towards pupils developing a sense of ownership of their own learning;

it's about getting them involved with what they're learning about. Instead of me telling them what they have to do [...] They have more ownership over it $[\ldots]$ that engages them a bit more I think.

Ms Abbot consistently provided opportunities for pupils to lead their own learning, and to develop a sense of ownership of their own thinking. One way that pupil-led learning was supported in Ms Abbot's class was through the introduction of learning logs themselves. On introduction, Ms Abbot emphasised to pupils that logs were personal to each pupil and were not to be graded alongside other classroom work;

it's a bit like keeping a diary - I'm not going to check them, it's for you. I'm not going to mark them, sometimes I might look through them, sometimes I might ask you what you have written, sometimes you might show what you have written to your friend, sometimes you can share it with the class.

This excerpt from pupils' introduction of learning logs emphasises that they are intended to be something personal for pupils with no 'right or wrong' answers - thus reinforcing their use as a tool for individuals to think about their own thinking.

Overall, we found that the context for our investigation of STAs was an environment that clearly valued pupils' perspectives of their own thinking and learning, in addition to nurturing 
pupils to persist and show resilience in their lessons. Ms Abbot repeatedly provided opportunities for pupils to share their perspectives about their thinking and learning in a classroom that offered a safe and nurturing atmosphere. Such an approach is consistent with literature that has emphasised characteristics of the learning environment as conducive to metacognition, including the avoidance of direct comparisons between pupils, not forcing pupils to reveal their lack of understanding publicly, and precluding embarrassment or excessive frustration (Robson, 2010).

\section{Pupil engagement with Structured Thinking Activities}

Despite Ms Abbot's success in creating a nurturing, safe environment in which to explore thinking and learning, we found that pupils were often unmotivated to engage with STAs. Pupils' lack of motivation was particularly evident at the beginning of the school year, however it continued to be a theme throughout data collection. When learning logs were initially described to the class, there was explicit resistance from some pupils, with some commenting to the teacher that they did not wish to take part. Regardless, as the following observed teacher-pupil learning log discussion highlights, pupils were given no choice about the requirement to engage with the end of week STAs;

John: I don't want to do this!

Ms Abbot: Well, we're all going to do it, and if you don't want to do it, then I can give you some extra work to do.

Here, Ms Abbot's response that the choice for pupils is either to complete STAs or 'extra work' suggests that the activities are compulsory, despite a focus on pupil-led learning and personalisation. Whilst Ms Abbot's response may reflect a broad strategy for dealing with dissent and disengagement rather than being specifically tied to the use of STAs, the insistence that pupils have to engage with metacognitive tasks is inherently contrary to the principle behind them, namely that pupils should be responsible for their own learning.

The lack of motivation towards STAs was also made clear during unstructured interview with the researcher, with a pupil describing learning logs as tiring activities when 'all we do is sit on chairs and write the boring stuff'. The activities were also perceived as tiring; 'I sometimes think that um, it's a wee bit, like, a wee bit tiring because we have to sit down and do work in silence, because the rest of the class are like 'aaarghh' [makes loud noise and gesticulates]'. As the above excerpts suggest, pupils were often openly reluctant to take part in STAs, describing them as boring.

Another aspect of timing that presents a clear barrier to engagement is the positioning of STAs alongside other tasks. Indeed, Friday afternoons were often described by Ms Abbot in discussion with pupils as a time to complete unfinished work from the week, 'Can I ask you nicely if you have not finished your writing this week, can you finish this off'. The conclusion that timing is an important factor was further reinforced in unstructured interview between the researcher and Ms Abbot in the spring term, when she described that STAs tended not to be completed during busy times, such as at the end of term; 'We didn't do it over Christmas, as you know, Christmas is quite manic, so we haven't done it since the $9^{\text {th }}$ [of December]'.

In sum, we interpret STAs as being placed at a times that devalue the importance of the activities themselves, creating the suggestion that STAs are a final piece of work to be completed at the end of the week, after plan and before relaxation. As a result, the timing of activities acted to marginalise the STAs themselves, providing the implication that the 
activities were not particularly important. Overall, therefore, the present findings strongly suggest that the timing of STAs is a potential barrier to pupil engagement, inhibiting their use as a tool for facilitating metacognition and providing an explanation for the lack of traction of STAs.

Pupil reflections focusing on describing topics of interest

As described above, at times we found that pupils indicated that they lacked motivation to take part in STAs. Furthermore, when pupils did reflect on their thinking and learning as part of STAs, we found that rather than engaging with the kind of detailed metacognitive reflection intended, pupils most commonly provided broad descriptions of topics learnt, particularly those that they found interesting or exciting. Such a focus on topics of interest was evident in analysis of pupils' written STAs, as shown in Figures 3 and 4.

[Figure 3 about here]

[Figure 4 about here]

Teacher-pupil interactions also provided insight about pupils' focus on topics. For example, in response to questions and sentences initiated by Ms Abbot in a observed class meet, we found that pupils repeatedly provided superficial responses that focused on topics rather than skills, and most commonly related to key areas of interest;

Ms Abbot: What else have you learned this week, Susan?

Susan: I learned in Assembly that girls are better spies than boys. Ms Abbot: Why are girls better spies than boys?

Susan: Because we can get changed faster

Ms Abbot: Something you have learned in class this week, Elsie?

Elsie: The $7 x$ tables, because I think the $7 x$ tables are really fun.

Later in the same observed discussion, pupils continued to refer to interesting topics, in this case the class project;

Ms Abbot: What would we like to learn more about next week? Derek?

Derek: Roman gods.

Ms Abbot: You would like to learn more about Romans? David?

David: Romans in general.

Here, we can see that pupils responded to teacher prompts by stating topics or events of interest. Discussion with the researcher in class during an unstructured researcher-pupil interview provided further instances of pupils focusing on aspects of lessons that they found most interesting;

Researcher: What are you looking forward to learning about next week?

Laura: I want to learn more about how the Roman emperors lived.

Researcher: How the Roman emperors lived? Why would you like to learn more about that?

Laura: Because, we know how roman soldiers lived, but we don't know about how roman emperors lived, even though I have a horrible history book about the Romans [a while later]

Researcher: Is there anything that you want to learn more about that is not to do with Romans?

Laura: Yes, I want to learn more about WW1, WW2, the battleships... 
This excerpt demonstrates that throughout STAs, Laura was clearly motivated to focus on lessons she was interested in. This is further evidence of our finding that in class meets, pupils most commonly referred to topics or 'facts' rather than skills or aspects of the thinking process. Across our observations, we found that pupils were motivated to talk about their thinking, but only to the point of providing very superficial reflections about topics of interest. Equally, the data demonstrate that pupils did not provide in-depth assessments of their learning, instead providing responses that focused on repetition of topics. As such, pupils tended to compound enjoyment with skill when taking part in discussions as part of STAs, and therefore, were limited in the extent that thought about their own cognition (a fundamental characteristic of metacognition, see Flavell, 1979; Schraw \& Moshman, 1995). Of course, in interpreting pupil engagement with the task, it is important to consider the ways in which the STAs were themselves structured. In the above example, the STA was presented to pupils through a prompt that explicitly encouraged students to think about what they were looking forward to in the week ahead. This finding emphasises that a clear influence upon students' engagement with STAs was the very content of the STA itself - students may therefore have compounded enjoyment with skill because (at least in part), the STA prompts themselves did not clearly distinguish between enjoyment and skill. This finding is reminiscent of the work of Desoete (2008), who described the close association between the tools used to measure metacognition and the insights into metacognition these provide - "how you test is what you get" (p204).

\section{Teacher-Pupil interactions and metacognition}

Thus far, we have described that despite reflections on thinking and learning being explicitly valued by Ms Abbot, pupils were often poorly motivated to take part in STAs. Moreover, in practice, the output produced using STAs often focused upon superficial aspects of thinking and learning, such as describing learnt topics of interest. Given these themes, a key finding in our research is the critical role of teacher-pupil interactions in eliciting metacognition from pupils. To be clear, we consistently observed that during discussions (that occurred as part of the STAs), Ms Abbot encouraged pupils to go beyond their initial superficial reflections. In particular, by asking elaborative questions and explicitly voicing her own cognitions, Ms Abbot was able to use discussions as a way to 'scaffold' pupil metacognition.

\section{Encouraging elaboration - introducing the language of learning}

Ms Abbot used speech to encourage elaboration by providing examples of the kind of response that she expected. In this sense, Ms Abbot elaborated for pupils, providing additional information or examples about the ways that pupils might think about and manage their own thinking. In these episodes of elaboration, Ms Abbot often described skills and made links between pupils' responses and the skills that they had developed. Often, Ms Abbot elaborated in short, passing comments in response to pupils' broad reflections during observed class meets, such as 'we learnt to code. So, we learnt little new things, but we also went deeper into things, like we went deeper into coding, didn't we?'. In addition, Ms Abbot explicitly elaborated during STA discussions. In the first observed class meet at the beginning of the school year, Ms Abbot supported pupils' goal-setting through description and elaborated discussion of the target-setting process;

Think of a goal that you have for next week. Remember we have our p4 goals for the year [on board]. Think about something you can set yourself for next week. Just something that you actually think you can get done in a week. So, it can't be something like, "eh, next week my goal will be that I will learn to be an astronaut, and fly to Mars, and set up a colony on another planet" 
[laughs]. Will I manage that in one week? Maybe if I try really hard, then in ten years. Something that you would really, really like to either get better at, to improve on for next week?

Here, Ms Abbot described what an unrealistic goal would look like. Using a humorous example, Ms Abbot encouraged pupils to set a realistically achievable goal for a week. As such, Ms Abbot made the process of goal-setting explicit to pupils and used an example to demonstrate what a 'useful' goal might look like (or in this case, not look like). Moreover, when pupils responded with goals, such as "at gymnastics, doing the pummel", Ms Abbot used questioning to encourage deeper responses from pupils, "and how do you think you can get better at that?" Therefore, in this interaction, Ms Abbot continually paired modelling with questioning as a way to promote pupils' metacognitive thinking.

Later in the same discussion between teacher and pupils, Ms Abbot again elaborated on the process of thinking about thinking, by describing how Laura's goal might be achieved;

Laura: [My goal] is to speak at least three sentences in fluent French Ms Abbot: Oh, now I think we can do one already, 'cause we have done one this week haven't we? You want to learn three? So next week, will we try to do maybe a sentence on Monday, and then a sentence on Thursday, and then by Friday, we could maybe try to fit them all together.

Here, by elaborating on Laura's goal and linking to goals already achieved, Ms Abbot provided an example for pupils of how they might plan to achieve a goal. In this example, Ms Abbot extended upon Laura's response, and it is of note that Laura did not respond to the elaboration provided by Ms Abbot - with the teacher effectively demonstrating for Laura how a plan might be created to achieve a goal. Clearly, therefore, at the beginning of the school year Ms Abbot played a key role in supporting pupil metacognition during STA discussions. In this respect our findings illustrate that teachers can encourage metacognitive reflection by asking pupils to 'go deeper' through questioning, as well as by providing examples of the ways that pupils could themselves think about and manage their own thinking, by making the internal process of metacognition explicit for pupils through modelling. The pedagogical approach of modelling has indeed been identified as important for the facilitation of metacognition; uncovering internal mental processing and providing pupils with an example from which they can model their own metacognition (Wall \& Hall, 2016). Importantly, the observations presented here strongly suggest that the teacher is not solely providing a metacognitive account of their own cognitions (i.e., showing that they are metacognitive), but is instead effectively showing how pupils could themselves be more metacognitive in their own reflections.

\section{Striking the Balance}

Clearly, where pupils provided relatively superficial metacognitive reflections, Ms Abbot played a key role in describing for pupils, what metacognition might look like. Importantly, we also observed instances where the teacher used closed questions or would evaluate for pupils (often in an effort to praise). For example, when reflecting on targets in the spring term as part of an observed teacher-pupil discussion surrounding achievement logs, Ms Abbot asked pupils to reflect using 'thumbs'; 'Thumbs up if you think you've achieved, thumbs to the side if you think that you kind of have achieved but you need to revisit it, or thumbs down if you think you've missed it'. Ms Abbot continued to structure pupils' evaluations by reading aloud targets from the achievement log and asking pupils to evaluate by showing their thumbs; 
Writing. I can use a range of connectives to join and extend my sentences. We've kind of done that haven't we? But we need a wee bit more work, a bit more help on extending our sentences. We'll do that next week [...] What about improving our creative writing skills? That should be a big thumbs up from everyone [...] Free writing. Do you enjoy free writing? [yeah]. And I think it's really helped us with our creative writing skills, because it's helped our imagination. So, I think we are a big green for that one.

In this example, the method of evaluation used by Ms Abbot was to ask pupils to indicate with thumbs whether or not targets had been achieved. The reflection elicited was in the form of a yes/no evaluation, rather than a reflection on the quality or process of learning. Furthermore, Ms Abbot then proceeded to effectively evaluate for pupils, demonstrating a distinction between modelling one's own metacognition, and making the metacognitive process explicit for pupils. Importantly, therefore, whilst the talk of teachers is critical for scaffolding pupils' developing metacognition, at times it may also restrict pupils' opportunities for individual (metacognitive) reflection.

In another observed discussion when evaluating targets in the achievement logs, Ms Abbot again provided guidance about how pupils should evaluate their targets;

The next bit is our key learning targets from term 3, from when we did the Romans. We talked about them last week, I want you to go through them and traffic light the circles. I'll tell you right now that no-one should be red for any of them, because everyone has taken part in all of them.

In this excerpt, Ms Abbot again directed pupils about their targets, stating that no one should have red evaluations as they all took part. Here, we see that a delicate balance exists in providing structure and support for the class to reflect, whilst encouraging pupils to independently reflect on their own thinking. Thus, there are opportunities to encourage metacognition in even the most common of classroom practices (a point that we follow up in more detail in the discussion).

\section{Encouraging elaboration from pupils}

Thus far, we have found several aspects of the classroom environment that influence pupils' engagement with STAs, and more specifically, the critical role of the teacher in encouraging metacognition through STAs. A key finding of the present study is that the best examples of pupils thinking about their thinking (i.e., engaging in metacognition) throughout STAs was through interaction between teacher and pupils. As outlined above, we found that Ms Abbot played a critical role in encouraging elaboration from pupils in relation to their descriptions of their own thinking and learning; particularly in response to pupils' broad or superficial responses. Ms Abbot consistently encouraged pupils to themselves elaborate on their responses during class meets, with her requests becoming more explicit as the year progressed. At the beginning of the year, Ms Abbot was observed to introduce the idea of elaborating on responses in a non-threatening manner, by stating that pupils could choose whether they would like to elaborate on their responses during the activity;

If you really want to, you can go, "I’ve enjoyed reading because...". You don't need to give the reason, but you can give the reason if you want to share it. The next sentence is: something I've found tricky this week has been. Now something you've found tricky - this can be something you have learned that's been tricky. So, you might say this week I have found maths tricky, 
and you might say I have found maths tricky because, or you can just leave it like that.

Here, Ms Abbot encouraged (but did not insist) that pupils elaborated on their answers, by stating why they provided their response (i.e., providing justification for their response). As such, Ms Abbot used interaction to encourage pupils to participate in their learning, and critically, to move from superficial reflections to more metacognitive reflections (i.e., cognitions about cognitions), whilst maintaining an environment that was safe and nonthreatening (Schraw, 1998).

Ms Abbot also encouraged pupils to think about strategies during class meets. An example is evident in an observed discussion between Ms Abbot and Amy in a class meet about times tables;

Ms Abbot: You have tried very well with your times tables this week. What things have we used to learn the times tables this week?

Amy: Em, we have used the triangles and the [inaudible]

Ms Abbot: What did the triangles help us to remember, which times table?

Amy: Three.

Ms Abbot: Three, and what about the five pence?

Amy: Five

Ms Abbot: The five. And we made posters, and we did lots of different tricks, didn't we?

Amy: Yep.

In a later observed teacher-pupil discussion, Ms Abbot again encouraged pupils to think about strategies used in relation to times tables, asking, 'Have we worked hard at our times tables this week? [Pupils say yes] What helps you learn them Sarah, is it like a strategy or a technique?'. Here we see that Ms Abbot used questioning to encourage pupils to reflect on the strategies used in numeracy, and to compare between different strategies for different times tables. As such, Ms Abbot brought pupils' thinking process to the surface by focusing on the strategies used and explicitly elaborating on pupils' initial (and often relatively superficial) responses. In doing so, evidence suggests that the STAs employed in this class could be seen as a pedagogical tool that was catalytic of metacognition through interaction (Baumfield et al., 2009).

Whilst at the beginning of the year Ms Abbot often provided examples and elaborated on skills for pupils, as the year progressed she insisted more that pupils themselves reflect on the process of task and the skills involved in classroom topics;

Ms Abbot: Does anyone want to tell me something that they have learned this week, anything they have learned this week?

Laura: That the girls make better Romans than the boys

Ms Abbot: So, why do the girls make better Romans than the boys?

Laura: Because when we were supposed to compose ourselves, the girls got together instantly, and the boys were like 'aahhhhh' [flaps arms around]

Ms Abbot: So, what skills did the girls use that the boys didn't?

Laura: Listening.

In this excerpt, we are again reminded of the tendency for pupils to refer to general skills and events that they found interesting. In response, Ms Abbot used questioning to steer the conversation back to the underlying skills. 
As the school year progressed, the critical role of Ms Abbot in persistently supporting pupils' metacognition throughout STAs was evident. In a review of term learning, Ms Abbot was observed to ask pupils to elaborate on the skills learnt in topic work, asking pupils, 'What kinds of things have we been learning about in Romans? Not about things, but about how to do things?' In response, pupils began to discuss skills more, however the following excerpt demonstrates that pupils still often tended to revert back to focusing on topics;
Ali: How to research?
Ms Abbot: How to research, what have we been learning about how to research things?
Ali: Um, like primary and secondary $[\ldots]$
Ms Abbot: What else have we been learning about with the Romans, Laura?
Laura: How to work in the Roman army
Ms Abbot: how to work, how they lived. We've been comparing how they lived to how we live, haven't we? Because we've been comparing, can anyone tell me any similarities or differences between the way they lived and the way we live.

Here, in response to Ms Abbot's question about how to do things, Ali responded by describing a skill that has been explicitly described as part of the class project, namely research. As the conversation progressed, we see that Laura reverted back to the recall of information, using the 'how' term in a way that allowed her to recall interesting information about the class project. In response, Ms Abbot again steered the conversation to focus on the underlying skill of comparison.

In an observed discussion around achievement logs, Ms Abbot explicitly made a differentiation between describing topics and describing skills when evaluating pupils' strengths in maths, asking;
Ms Abbot: What specifically in maths? Working your best in maths? Trying hard in maths? Having a determined attitude in maths? Times tables? Problem- solving? Just maths in general? [Paul nods]
Karl: I'm good at PE and maths
Maida: PE
David: PE, maths...
Ms Abbot: Now you're all just naming me subjects, so what is it that you're good at, is it using the skills, is it working together as a team? Is it good spatial awareness, what is the skill that you're good at?
Donald: Em... coding.

This example is typical of Ms Abbot's use of questioning to attempt to 'dig deeper' than superficial naming of subjects. Pupils' tendency to provide superficial reflections is clear, and we see the critical role of the teacher in balancing between providing more detailed elaborations herself, and allowing pupils the freedom to elaborate themselves. The above excerpts, therefore, demonstrate the dual role of the teacher in providing enough support to allow pupils to 'see' the ways that one can think about thinking, whilst also encouraging pupils to themselves think about their thinking.

\section{Discussion}

The present research aimed to investigate the ways that pupils think about and manage their own thinking (i.e., engage with metacognition) through STAs. Using observational methods triangulated across participant observation, interviews and written outputs, we were able to 
reveal the fundamental role of teacher-pupil interactions in facilitating metacognition. Ms Abbot, consistently emphasised the importance of effort and persistence, sought pupils' perspectives about thinking and learning, and used these perspectives to plan lessons. Nonetheless, despite the fact that the learning environment was clearly supportive of metacognition, we found that pupils were often unmotivated to take part in STAs, and when they did, they most often provided superficial responses about their thinking and learning. Moreover, timing was highlighted as an aspect of STAs that could act as a barrier to metacognition, because STAs were typically held at times when pupils were particularly tired or unmotivated, which acted to marginalise the activities themselves. From this perspective, the focus by pupils upon topics of interest can be understood as an example of the idea that "how you test is what you get" (Desoete, 2008, p204), highlighting the close association between the tools used to elicit metacognition and the insights into metacognition these tools can provide (Desoete, 2008; Gascoine et al., 2017).

Most importantly for the present analysis, the present findings revealed clear instances where metacognition could be observed, in particular during interactions between the teacher and pupils as part of class meets. Overall, therefore, our key finding is that teacher-pupil interactions play a critical role in supporting metacognition, through elaboration and questioning as part of STAs. As such, our findings point to the critical importance of the class teacher for encouraging metacognition and suggest that the very value of STAs is in providing a platform for teacher-pupil interactions.

A clear outcome from our analysis of STAs was the role of teacher talk in creating a classroom environment that was facilitative of metacognition. Ms Abbot clearly placed value upon pupils' perspectives about their own thinking and learning. Prominent classroom approaches (including growth mindsets and pupil-led learning) were used to place explicit value upon affective attributes such as persistence, whilst also clearly valuing pupils' own perspectives about their thinking and learning. Recent research suggests that the efficacy of approaches such as growth mindsets may have been over-emphasised (e.g., see Sisk et al., 2018). Nonetheless, such approaches remain useful for creating a classroom culture that is supportive of metacognition by making it clear that pupils' perspectives are valued (Schraw, 1998; Uluduzli \& Gunbayi, 2018). Critically, however, in the case study presented here we found that to promote metacognition more was needed than the creation of a supportive classroom culture: teacher-pupil interactions were necessary to facilitate metacognition.

\section{Exploring Metacognition through STAs}

The observations presented in this study provide clear insights for developing understanding about metacognition in the applied context of the classroom. In the primary four classroom examined here we found that indicators of metacognition were often superficial, with pupils providing broad reflections on topic or tasks of interest. The apparent lack of metacognitive engagement is particularly clear in the written outputs produced by pupils (as illustrated in Figures 1-4). One important theoretical implication of this finding is that any attempt to assess metacognition based on the output of STAs alone would most likely lead to the conclusion that metacognition is not really present within the primary school classroom, providing further support for the wider claim that 'how you test is what you get' (Desoete, 2008, p204). Importantly, however, a clear distinction could be made between the broad and procedural reflections provided in written STAs, with the more in-depth reflections on cognition elicited through teacher-pupil discussion. By observing the content of teacher talk, and pupil's responses to it, we were able to provide strong evidence of metacognition that went well beyond what was produced in the written STAs. Thus, overall, our findings reveal 
that the middle primary school years are critical for pupils' developing metacognition, but this is only evident when engagement with STAs in understood within the wider social context of teacher-pupil interactions.

Where pupils struggled to metacognitively reflect on the process of thinking, we saw clear examples of Ms Abbot being responsive to pupils' struggles, providing additional support by using metacognitive vocabulary and making the language of thinking explicit. From this perspective, one reason that there was little evidence of metacognition within the written STAs may be that pupils had not yet learnt the language of metacognition. As outlined in our results, in some instances, Ms Abbot ultimately 'filled in the gaps' by elaborating on pupils' reflections herself and making the thinking process visible through modelling. To be clear, our observations revealed that the teacher often provided support by demonstrating metacognition for pupils (i.e., generating examples of metacognition that pupils might in future produce for themselves), rather than by showing the pupils her own metacognitive reflection (i.e., acting as a role model that could be copied). In broad terms the benefits of modelling are well understood - with research demonstrating the effectiveness of teachers being 'metacognitive role models' for supporting pupils' developing metacognition (Wall \& Hall, 2016). The present findings are novel, however, in suggesting that the teacher's role is not simply to act in a metacognitive way themselves, but rather to show pupils what their metacognition might look like.

Our finding of the critical role of teacher talk demonstrated that Ms Abbot continuously negotiated pupils' engagement of metacognition by providing scaffolding sensitive to the needs of pupils (Vygotsky, 1978; Wood, Bruner \& Ross, 1976). Such an understanding emphasises the socio-cultural, relational aspects of metacognitive development. Whilst educational approaches focused on reflection and development were valuable for creating a foundation for pupils to share their thinking about thinking, we found that these approaches alone were not sufficient for encouraging metacognition via STAs. To be clear, we found that teachers facilitated pupil metacognition through direct teacher-pupil interaction about pupils' own thinking processes. As such, our findings highlight that metacognitive approaches are complimentary, yet distinct, from other existing classroom approaches (such as growth mindsets).

\section{STAs as Pedagogical Tools for Metacognition}

Our findings emphasise the multiple factors that underpin teachers' ability to support metacognition using STAs. For example, we highlight the influence of teachers developing a classroom environment that values pupils' perspectives of their own thinking and advocates affective attributes such as persistence. By contrast, our findings regarding the timing of STAs highlights the challenge that teachers face in trying to facilitate pupil metacognition within the already demanding classroom routine. In addition, we demonstrate the value of pupils being equipped to independently think about and manage their thinking by firstly seeing how people can think about and manage their thinking before they can do it themselves. As such, our findings support the view that teachers must make the thinking process explicit, by making the language of metacognition visible, by modelling, and acting as a 'metacognitive role model' (Dignath et al., 2008; Wall \& Hall, 2016). Ultimately, teachers must provide opportunities for pupils to independently think about and manage their own thinking.

One important outcome of the present findings is the clear demonstration that teachers must play a dual role - alternating between showing pupils what metacognition looks like (by modelling metacognition in response to pupils' initial reflections - which were often 
relatively superficial) and encouraging pupils to think about thinking themselves though elaborative questioning. That is, at times we found a clear tension between pupils providing personal reflections, versus the teacher structuring or guiding reflections for pupils. The forms and functions of modelling is something to be critically reflective of given the findings of the present research. We found that teachers can not only model metacognition by themselves being metacognitive and making this explicit (Wall \& Hall, 2016), but they can also model metacognition by showing pupils how they can set goals, plan or reflect in relation to pupils' own reflections (by voicing examples of metacognition for pupils). Whilst these different forms of modelling may each be thought of as important pedagogical tools within a teachers' repertoire to facilitate metacognition throughout STAs, the tension between modelling and individual reflection becomes apparent. That is, where pupils are encouraged to respond in a particular manner (for example, how to achieve a goal, or to what extend that goal has been achieved), this may contrast with the notion of individual reflection. At first sight these aspects of teacher talk may appear to be contradictory to the aims of metacognition, however our evidence suggests that the variety of pedagogical tools that surround STAs are, in fact, precisely what makes STAs so important for promoting metacognition - pupils must be shown what their own metacognition could look like, before they can engage in metacognition themselves. From this perspective STAs provide a route for teachers to provide scaffolding for pupils' developing metacognition within their 'Zones of Proximal Development' (Vygotsky, 1978) - progressing from more teacher-focused instructional forms of interaction, towards more pupil-led reflections. Within this context, the present findings emphasise the catalytic nature of STAs for metacognition (Baumfield et al., 2009).

Given the multiple roles that we have identified to support pupil metacognition, our findings emphasise the fundamental importance of supporting teachers to develop practices to facilitate pupil metacognition through STAs. To be most effective, emphasis should be placed upon developing understanding about the value of interaction and elaborative questioning for the facilitation of metacognition, for example through explicit focus in initial teacher education and professional development, and more widely, via educational policy. In addition, our view is that there must be greater acknowledgement that whilst metacognitive approaches may ultimately develop more independent learners, supporting metacognitive development requires a significant commitment by teachers. Rather than being a 'quick fix', metacognition requires lengthy and relational support across time, as well as requiring considerable skill from teachers in navigating the dual role that is required to support metacognitive development - alternating sensitively between asking metacognitive questions and giving metacognitive answers, as pupils struggle to learn how to express their own metacognition.

In summary, we found that rather than the existence of STAs being evidence in themselves of metacognition in the classroom, in fact STAs form only the basis for beginning to understand how metacognition can be encouraged within classrooms. Indeed, the present findings highlight the complexity of encouraging metacognition within the classroom environment, with factors such as the classroom culture and the timing of activities clearly influencing pupil engagement. More importantly, alongside these factors, we find that the most important influence on metacognition is the talk of the teacher in discussion throughout STAs. Indeed, we found that metacognition only really occurred when teachers used modelling and elaborative questioning to elicit deep reflections about the process of thinking and learning. Our findings also highlight clear avenues for future research. For example, the dual-role nature of teacher talk suggests the need to understand metacognitive development as an iterative and interactive process, suggesting that the process of metacognitive learning from 
one task to another requires further investigation. The demonstration that teacher talk is essential for metacognitive development in the classroom suggests that future studies should focus on less on the written outputs of STAs themselves, and more on understanding the critical features of effective teacher-pupil interactions that surround them. 


\section{References}

Authors (2019)

Author (2019)

Adler, P. A. \& Adler, P. (1998) Observational Techniques. In Denzin, N.K. and Lincoln, Y.S. (Eds). Collecting and Interpreting Qualitative Materials (pp 79-109). London: Sage

Audet, R. H., Hickman, P., \& Dobrynina, G. (1996). Learning logs: a classroom practice for enhancing scientific sense making. Journal of Research in Science Teaching, 33(2), 205-222.

Barclay, J. (1996). Learning from experience with learning logs. Journal of Management Development, 15(6), 28-43. DOI:10.1108/02621719610120129

Baumfield, V. M., Hall, E., Higgins, S., \& Wall, K. (2009). Catalytic tools: understanding the interaction of enquiry and feedback in teachers' learning. European Journal of Teacher Education, 32(4), 423-435. doi:10.1080/02619760903005815.

Braun, V., \& Clarke, V. (2006). Using thematic analysis in psychology. Qualitative Research in Psychology, 3(2), 77-101. doi:10.1191/1478088706qp063oa.

Brown, A. L. (1987). Metacognition, executive control, self-regulation, and other more mysterious mechanisms. In Weinert, F., \& Kluwe, R. (Eds.). Metacognition, Motivation, and Understanding (pp. 65-116). New Jersey: Erlbaum.

Craft, A., Cremin, T., Hay, P., \& Clack, J. (2014). Creative primary schools: developing and maintaining pedagogy for creativity. Ethnography and Education, 9(1), 16-34. doi:10.1080/17457823.2013.828474.

Desoete, A. (2008). Multi-method assessment of metacognitive skills in elementary school children: how you test is what you get. Metacognition Learning, 3(3), 189-206. doi:10.1007/s11409-008-9026-0.

Dignath, C., Buettner, G. \& Langfeldt, H. P. (2008). How can primary school students learn self-regulated learning strategies most effectively? A meta-analysis on self-regulation training programmes. Educational Research Review, 3(2), 101-129. doi:10.1016/j.edurev.2008.02.003

Flavell, J. H. (1976). Metacognitive Aspects of Problem Solving. In Resnick, L.B. (Eds.). The Nature of Intelligence (pp. 231-235). London: John Wiley \& Sons.

Flavell, J. H. (1979). Metacognition and cognitive monitoring: A new area of cognitivedevelopmental inquiry. American Psychologist, 34(10), 906. doi:10.1037/0003066X.34.10.906.

Gascoine, L., Higgins, S., \& Wall, K. (2017). The assessment of metacognition in children aged 4-16 years: a systematic review. Review of Education, 5, 3-57. doi:10.1002/rev3.3077.

Geertz, C. (1973). The Interpretation of Cultures. New York: Basic Books.

Hacker, D. J., \& Dunlosky, J. (2003). Not all metacognition is created equal. New Directions for Teaching and Learning, 95, 73-79. doi:10.1002/tl.116. 
Hammersley, M. (2006). Ethnography: problems and prospects, Ethnography and Education, 1(1), 3-14. doi:10.1080/17457820500512697.Higgins, S. E., Baumfield, V. M., Lin, M., Moseley, D., Butterworth, M., Downey, G., Gregson, M., Oberski, I., Rochett, M., \& Thacker, D. (2004). Thinking Skills Approaches to Effective Teaching and Learning. London: Social Science Research Unit, University of London.

Higgins, S., Katsipataki, M., Villanueva-Aguilera, A.B., Coleman, R., Henderson, P., Major, L.E., Coe, R. \& Mason, D. (2016) The Sutton Trust-Education Endowment Foundation Teaching and Learning Toolkit Manual. Education Endowment Foundation, London.

Higgins, S., Wall, K., Baumfield, V., Hall, E., Leat, D., Woolner, P., ... \& Lofthouse, R. (2006). Learning to learn in schools phase 3 evaluation: Year two report. Newcastle: Campaign for Learning.

Higgins, S.,Wall, K., Baumfield, V. M., Hall, E., Leat, D., Moseley, D., \& Woolner, P. (2007). Learning to Learn in Schools Phase 3 Evaluation: Final Report. London: Campaign for Learning.

Hurst, B. (2005). My journey with learning logs. Journal of Adolescent and Adult Literacy, 49:1, 42-46. doi:10.1598/JAAL.49.1.5

Kidner, C. (2013). SPICe Briefing: Curriculum for Excellence. Edinburgh: Scottish Parliament.

Kleinsasser, A. M. \& Horsch, E. A. (1992). Teaching, assessment, and learning: Invitation to a discussion. ERIC Document Reproduction Service No. ED 352419.

Lewis, A., \& Porter, J. (2007). Research and Pupil Voice. In Florian, L. (Ed.) Handbook of Special Education (pp. 222-232). London: Sage.

Mallozzi, F. N. \& Heilbronner, N. (2013). The effects of using interactive student notebooks and specific written feedback on seventh grade students' science process skills. Electronic Journal of Science Education, 17(3), 1-24.

McCrindle, A. R. \& Christensen, C. A. (1995). The impact of learning journals on metacognitive and cognitive processes and learning performance. Learning and instruction, 5(2), 167-185. doi:10.1016/0959-4752(95)00010-Z.

Moon, J. (2002). Learning logs: A Handbook for Academics, Students and Professional Development. London: Kogan Page Limited

Paris, S. and Paris, A. (2001). Classroom applications of research on self-regulated learning. Educational Psychologist, 36(2), 89-101. doi: 10.1207/S15326985EP3602_4

Perry, N. E. (1998). Young children's self-regulated learning and contexts that support it. Journal of Educational Psychology, 90(4), 715-729. doi:10.1037/0022-0663.90.4.715.

Perry, N., VandeKamp, K., Mercer, L., \& Nordby, C. (2002). Investigating teacher-student interactions that foster self-regulated learning. Educational Psychologist, 37(1), 5-15. doi:10.1207/S15326985EP3701_2.

Ritchhart, R., Turner, T., \& Hadar, L. (2009). Uncovering students' thinking about thinking using concept maps. Metacognition and Learning, 4(2), 145-159.

doi:10.1007/s11409-009-9040-x. 
Roebers, C.M. (2014). Children's Deliberate Memory Development: The Contribution of Strategies and Metacognitive Processes. In Bauer, P.J. \& Robyn Fivush, R. (Eds). The Wiley Handbook on the Development of Children's Memory (pp 865-894). Oxford: Wiley-Blackwell.

Roebers, C. M., Schmid, C. \& Roderer, T. (2009). Metacognitive monitoring and control processes involved in primary school children's test performance. British Journal of Educational Psychology, 79(4), 749-767. doi: 10.1348/978185409X429842

Robson, C. (2011). Real World Research (Third Editon). West Sussex: Wiley

Robson, S. (2010). Self-regulation and metacognition in young children's self-initiated play and reflective dialogue. International Journal of Early Years Education, 18(3), 227241. doi:10.1080/09669760.2010.521298.

Schraw, G. (1998). Promoting general metacognitive awareness. Instructional Science, 26(1), 113-125. doi: 10.1023/A:1003044231033

Schraw, G. \& Moshman, D. (1995). Metacognitive theories. Educational Psychology Review, 7(4), 351-371.

Scott, B. M., \& Levy, M. G. (2013). Metacognition: Examining the components of a fuzzy concept. Educational Research eJournal, 2(2), 120-131. doi:10.1.1.1021.9065.

Sisk, V. F., Burgoyne, A. P., Sun, J., Butler, J. L., \& Macnamara, B. N. (2018). To what extent and under which circumstances are growth mind-sets important to academic achievement? Two meta-analyses. Psychological Science, 29(4), 549-571.

Smith, K.S., Rook, J.E. \& Smith, T.W. (2007). Increasing student engagement using effective and metacognitive writing strategies in content areas. Preventing School Failure: Alternative Education for Children and Youth, 51(3), 43-48. doi:10.3200/PSFL.51.3.43-48

Uluduz, H. \& Gunbayi, I. (2018). Growth mindset in the classroom. European Journal of Education Studies, 1(1), 173-186.

Vygotsky, L. S. (1978). Mind in Society: The Development of Higher Psychological Processes. Cambridge: Harvard University Press.

Wall, K. (2008). Understanding metacognition through the use of pupil views templates: Pupil views of Learning to Learn. Thinking Skills and Creativity, 3(1), 23-33. doi:10.1016/j.tsc.2008.03.004.

Wall, K., \& Hall, E. (2016). Teachers as metacognitive role models. European Journal of Teacher Education, 39(4), 403-418. doi:10.1080/02619768.2016.1212834.

Wall, K., \& Higgins, S. (2006). Facilitating metacognitive talk: a research and learning tool. International Journal of Research \& Method in Education, 29(1), 39-53. doi: 10.1080/01406720500537353.

Wall, K., Higgins, S., Remedios, R., Rafferty, V., \& Tiplady, L. (2012). Comparing Analysis Frames for Visual Data Sets: Using Pupil Views Templates to Explore Perspectives of Learning. Journal of Mixed Methods Research, 7(1), 22-42. doi:10.1177/1558689812450211. 
Wood, D., Bruner, J. S., \& Ross, G. (1976). The role of tutoring in problem solving. Journal of Child Psychology and Psychiatry, 17(2), 89-100. doi:10.1111/j.14697610.1976.tb00381.x. 